\title{
Diseño de un Sistema de Gestión para la Supervisión e Identificación de equipos médicos por medio de tecnología RFID en hospitales
}

\section{Design of a Management System for Monitoring and Identification of medical equipment using RFID in the hospitals}

\author{
Javier Humberto Rubio Plazas ${ }^{1}$ y Javier Mauricio Rodríguez ${ }^{2}$
}

\begin{abstract}
Resumen
En este artículo se da a conocer un estudio, para hacer uso de tecnología RFID en el control y gestión de equipos médicos del Hospital Universitario Remando Moncaleano Perdomo E.S.E de la ciudad de Neiva (Huila- Colombia). Para su desarrollo, se realizó un modelo de prueba con un prototipo RFID de la empresa Ericel \& Detec Ingeniería, el DTR.10, para simular el funcionamiento y determinar las características óptimas para el sistema que se pudiese implementar en el hospital. Para ello fue necesario crear un software de aplicación en Builder $\mathrm{C}++$, para la interfaz de comunicación entre el lector y los tags RFID, el cual se encarga además de procesar los datos obtenidos del tag (el UID en este caso), con una base de datos en MySQL que permite relacionar los datos del tag con la información los equipos médicos. Además se diseñó una aplicación en lenguaje PHP, con el cual el usuario podrá administrar la información de la base de datos.

A partir de los resultados obtenidos del prototipo, se hizo una propuesta de diseño y de los dispositivos del sistema del hospital. De acuerdo a lo anterior, se determinó que los dispositivos RFID que operan bajo el protocolo ISO/IEC 15693, a frecuencia de $13.56 \mathrm{MHz}$, eran adecuados para el sistema del hospital, debido a las características técnicas y operativas que ofrecen sus fabricantes.
\end{abstract}

Palabras Clave: RFID; Prototipo; DTRJO; UID; Interfaz; Lector; Tag; Base de datos; Protocolo; MySQL; PHP.

\section{Abstract}

This article announces a study to make use of RFID technology in the control and management of medical equipment at Hospital Universitario Hernando Moncaleano Perdomo ESE of the city of Neiva (Huila - Colombia). For its development, was performed a model test of a prototype RFID from Ericel Detec \& Engineering company, DTR 10 to simulate the operation and determine the optimal characteristics for the system that could be implemented in the hospital. It was necessary to create a software application in Builder $\mathrm{C}++$, to the communication interface between the reader and RFID tags, which is also responsible for processing data from the tag (the UID in this case), with a base MySQL data that relates the tag data with medical equipment information respective. It was design an application in PHP, with which the user can manage of the database information.

From the results of the prototype, it was made a proposal designed and of the hospital system devices. According to the above, it was determined that RFID devices operating under the protocol ISO / IEC 15693, a frequency of $1356 \mathrm{MHz}$, were adequate for the hospital system, due to technical and operational features offered by its manufacturers.

Keywords: RFID; Prototype; DTRJ O; UID; Interface; Reader; Tag; Data base; Protocol; MySQL; PHP.

IIngeniero Electrónico, Docente Universidad Surcolombiana-Neiva. Av. Pastrana-Carrera 1. 


\section{Introducción}

RFID (Radio Frequency Identification) es una tecnología de identificación automática por radiofrecuencia, similar en cuanto a su aplicación a la tecnología de código de barras. Un sistema RFID se compone básicamente de un lector, su antena, etiquetas o tags (compuestos de un microchip y una antena), y el middleware, que es el software que gestiona todo el sistema RFID a nivel de hardware, proporcionando los medios de proceso y almacenamiento de datos.

RFID ha llegado a ser una de las tecnologías de comunicación que ha experimentado un crecimiento más acelerado y sostenido en los últimos tiempos. La posibilidad que ofrece la lectura a distancia de la información contenida en una etiqueta o tag, sin necesidad de contacto físico, junto con la capacidad para realizar múltiples lecturas y en algunos casos su escritura, de forma simultánea, ha abierto caminos a un conjunto muy extenso de aplicaciones en una gran variedad de ambientes, desde la trazabilidad y control de inventario, hasta la localización y seguimiento de personas y activos, o la seguridad en el control de acceso.

El uso de RFID en instalaciones hospitalarias puede conseguir aumentar la seguridad y la visibilidad tanto de personas como de activos dentro de un hospital. Las aplicaciones posibles son casi ilimitadas, y cualquier situación que se vive cotidianamente en el ámbito sanitario puede mejorarse por medio de la tecnología de identificación por radiofrecuencia.

El Hospital Good Shepherd Advocate en Estados Unidos, hizo uso de etiquetas RFID pasivas dela empresa Sovereign Tracking Systems, para el etiquetado de equipamiento móvil, para el control de inventario. Se ha informado una reducción a la mitad de los errores de inventario (Attaran, 2006).

Varios hospitales estadounidenses se asociaron a principios del año 2004 para realizar una prueba de etiquetas de eXI Wireless, perteneciente actualmente a Verichip Corporation. El objetivo era la protección, localización y seguimiento de equipamiento médico en los hospitales. El objetivo del proyecto fue probar el sistemaAsetrac20, con el objetivo de reducir los costes de pérdidas de equipamiento hospitalario y las pérdidas de tiempo del personal dedicado a las búsquedas del equipamiento extraviado. Para ello se fijaron etiquetas RFID al equipamiento, para hacer saltar una alarma siempre que un determinado equipo saliera de su perímetro asignado. El sistema Asetrac dispone de una interfaz gráfica donde se pueden mostrar planos del lugar de despliegue, ayudando así al personal en el seguimiento de los equipos (Portillo, 2008).

Un hospital de 500 camas en el sureste de Georgia (Estados Unidos), buscaba una mejor manera de rastrear la ubicación y utilización de los equipos móviles dentro del hospital. A partir de la implementación del sistema de identificación por radio frecuencia y localización en tiempo real (RTLS), la gestión de activos es más eficaz y más económica. El sistema fue instalado en marzo del 2010 y se identificaron 1.900 activos, entre ellos, equipos de infusión, dispositivos de compresión y equipos respiratorios. "Cuando vine por primera vez en noviembre de 2004, realmente no tenía una idea clara de lo que estaba pasando con nuestros activos", dice Al Hardy, el responsable de compras de bienes de capital y gestión de activos del hospital. "No podíamos rastrear su ubicación, el costo de mantenimiento y la tasa de utilización. Ahora, antes de reemplazar el equipo o comprar equipo adicional, podemos usar el RTLS para determinar la tasa de utilización, la cantidad de tiempo que se demoró en reparar un equipo, y su uso para determinar si realmente se necesita gastar ese dinero" (RFID Journal, 2010).

El objetivo de este estudio, es crear la propuesta para aplicar RFID, en la ayuda de los procesos de control y gestión de los equipos médicos del hospital, para reducir inconvenientes en la administración, tanto de los equipos como de su información, debido al gran número de estos dentro de la institución. Además, examinar la posibilidad de usar RFID como un sistema antirrobo, haciendo uso de los propios tags y empleando lectores de largoalcance. 


\section{Metodología}

2.1 Sistema de gestión de base de datos: Para llevar el registro de los equipos médicos del hospital, se dispuso de una base de datos. Una base de datos es un $11_{\text {almacén }} 11$ que permite guardar grandes cantidades de información, organizada en forma de tablas, para que pueda ser encontrada y utilizada fácilmente. Para el desarrolla de la base de datos, se empleó un sistema de gestión de base de datos, que es un tipo de software muy específico, dedicado a servir de interfaz entre la base de datos, el usuario y las aplicaciones que la utilizan, el cual se compone de un lenguaje de definición, de un lenguaje de manipulación y de un lenguaje de consulta de datos.

El sistema de gestión de base de datos utilizado en la aplicación del prototipo fue MySQL, que es de licencia libre. Para el diseño de la base de datos en MySQL, primero se identificó la información que contendría la base de datos, creando así el diccionario de datos, para lo cual se tuvo en cuenta los datos más comunes que se manejan en las hojas de vida y reportes de mantenimiento de los equipos médicos. A partir del diccionario de datos se creó el modelo entidad-relación, para finalmente diseñar la base de datos en lenguaje SQL usando para ello la aplicación de MySQL (Valade, 2004). En él se definen los campos, los tipos de datos, las relaciones y restricciones de cada una delas tablas que componen la base de datos.

2.2 Interfaz para la administración de la base de datos: Para administrar la base de datos de MySQL, se usan comandos en lenguaje SQL. Para facilitar al usuario su manejo, se diseñó una interfaz visual para gestionar la información que hay en ella, el cual se encargará de ejecutar los comandos SQL necesarios, sin que la persona tenga que preocuparse sobre su programación.

Para el desarrollo de la interfaz que se conecta con la base de datos MySQL, se hizo uso de PHP, que es un lenguaje de programación interpretado, diseñado originalmente para la creación de páginas web dinámicas. Para ejecutar los comandos de MySQL desde PHP (Valade, 2004), se utilizó el programa servidor APACHE (Engelschall, 2001).

2.3Lector RFID DTR 10: Este lector de la empresa Ericel \&Detec Ingeniería (Ericel, 2009), cumple con la norma estándar ISO/IEC 15693 y permite leer tags en el rango de alta frecuencia HF de $13.56 \mathrm{MHz}$. Incorpora la función de anticolisión, para poder leer varios tags simultáneamente y tiene un alcance de lectura máximo de $10 \mathrm{~cm}$, debido a la potencia dela antena $(200 \mathrm{~mW})$. Se conecta a un computador a través de interfaz RS-232.

2.4 Tag RI-117-112A-03: Este tag de la compañía de Texas Instruments (TEXAS, 2004), es compatible con el estándar ISO/IEC 15693. Contiene un único código identificador o UID, el cual relaciona el equipo que contiene el tag, con la información respectiva en la base de datos.

2.5 Protocolo ISO/IEC 15693: Debido a los numerosos comandos que rige el protocolo ISO/IEC 15693 (Ericel, 2009) para el desarrollo del modelo, el interés se centró en la lectura de los tags para obtener el UID, para lo cual se empleó el comando de modo inventario.

Para utilizar el modo inventario, al byte de comando del paquete de pregunta se le asigna el valor hexadecimal de O1 (Tabla 1). De esta manera, el lector está en capacidad de leer los tags que estén dentro del rango de alcance de su antena.

Tabla 1.Paquete de pregunta en modo inventario

\begin{tabular}{|c|c|c|c|c|c|c|}
\hline $\begin{array}{c}\text { Inicio trama } \\
\text { (SOF) }\end{array}$ & $\begin{array}{c}\text { Longitud } \\
\text { del paquete }\end{array}$ & $\begin{array}{c}\text { Dirección } \\
\text { del nodo }\end{array}$ & $\begin{array}{c}\text { Bandera } \\
\text { de pregunta }\end{array}$ & $\begin{array}{c}\text { Datos de } \\
\text { Comando }\end{array}$ & $\begin{array}{c}\text { Fin trama } \\
\text { (EOF) }\end{array}$ \\
\hline 02 & $0 \mathrm{~B} 00$ & 0000 & $8 \mathrm{D}$ & 01 & 0000 & $857 \mathrm{~A}$ \\
\hline
\end{tabular}

Una vez recibe el paquete de pregunta por parte del lector, el tag genera un paquete de respuesta (Tabla 2). Al recibir el paquete de respuesta, el lector revisa los campos "Comprobar datos" y "Datos de respuesta" (Tabla 3), para verificar si se presentó algún error en la transmisión. Igualmente ocurre lo mismo en el paquete de respuesta para múltiples tags, en el cual el lector emplea la función anticolisión. 
Tabla 1. Paquete de respuesta en modo inventario

\begin{tabular}{|c|c|c|c|c|c|c|c|}
\hline $\begin{array}{c}\text { Inicio trama } \\
\text { (SOF) }\end{array}$ & $\begin{array}{c}\text { Longitud } \\
\text { del paquete }\end{array}$ & $\begin{array}{c}\text { Dirección } \\
\text { del nodo }\end{array}$ & $\begin{array}{c}\text { Bandera de } \\
\text { respuesta }\end{array}$ & Comando & $\begin{array}{c}\text { Comprobar } \\
\text { datos }\end{array}$ & $\begin{array}{c}\text { Datos de } \\
\text { respuesta }\end{array}$ & $\begin{array}{c}\text { Fin trama } \\
\text { (EOF) }\end{array}$ \\
\hline 02 & 1700 & 0000 & 00 & 01 & 6 bytes & 8 bytes & B649 \\
\hline
\end{tabular}

Tabla 3. Comprobación y datos de respuesta del modo inventario

\begin{tabular}{|c|c|c|c|c|}
\hline & Comp & ardatos & & Datos de respuesta \\
\hline $\begin{array}{c}\text { Datos } \\
\text { válidos }\end{array}$ & $\begin{array}{c}\text { Banderas de } \\
\text { colisión }\end{array}$ & $\begin{array}{l}\text { Código } \\
\text { error }\end{array}$ & $\begin{array}{l}\text { Datos en } \\
\text { memoria }\end{array}$ & UID \\
\hline 0100 & 0000 & $\mathrm{OO}$ & $\mathrm{OO}$ & $003191 F 217000007 \mathrm{EO}$ \\
\hline
\end{tabular}

2.6 Interfaz Lector RFID - Base de Datos: La interacción entre el prototipo RFID y la base de datos, se llevó a cabo mediante un software de aplicación realizado en C++Builder 6. El diseño dela interfaz se dividió en 2partes:

a) El código para el lector RFID: se encarga de la comunicación entre el lector y el tag, que consiste en el envío y recepción de comandos por puerto RS-232, que el lector interpreta para realizar la acción respectiva. El paquete de datos obtenido por el lector, se descompone mediante un algoritmo, hasta obtener el UID del tag.

b) El código para la base de datos: que se encarga de procesar los datos obtenidos del tag con la base de datos de MySQL, para así obtener la información relacionada con el equipo que contenga el UID. Para emplear la base de datos MySQL junto con la aplicación de C++ Builder, se utilizó el conector de origen de base de datos de MySQL, para ejecutar las sentencias SQL definidas en el programa.

\section{Resultados}

3.1 Administración de la información: La base de datos de MySQL junto con la interfaz visual en PHP (Figura 1), facilita la gestión de la información delos equipos médicos, permitiendo la integridad y seguridad de los datos. Con la interfaz se maneja información relacionada con la hoja de vida de los equipos, permitiendo la consulta, el ingreso y la modificación de los datos.

Una característica sobresaliente de esta interfaz, consiste en que a cada equipo se asigna automáticamente un código interno, para identificar cada uno de ellos de forma única dentro de la base de datos. De este modo, cuando un tag sea asigna a un equipo, los datos del tag (en este caso el UID), se relaciona con el código interno del equipo.

La interfaz da la posibilidad de visualizar los datos de un equipo en formato PDF, para que el usuario tenga la posibilidad de imprimirlo y guardarlo de manera física. Otra característica importante de esta interfaz, es la facilidad y efectividad en la búsqueda de la información, en el cual se han definido filtros que permiten una interacción y establecer mejor la comunicación de la interfaz con la base de datos. 


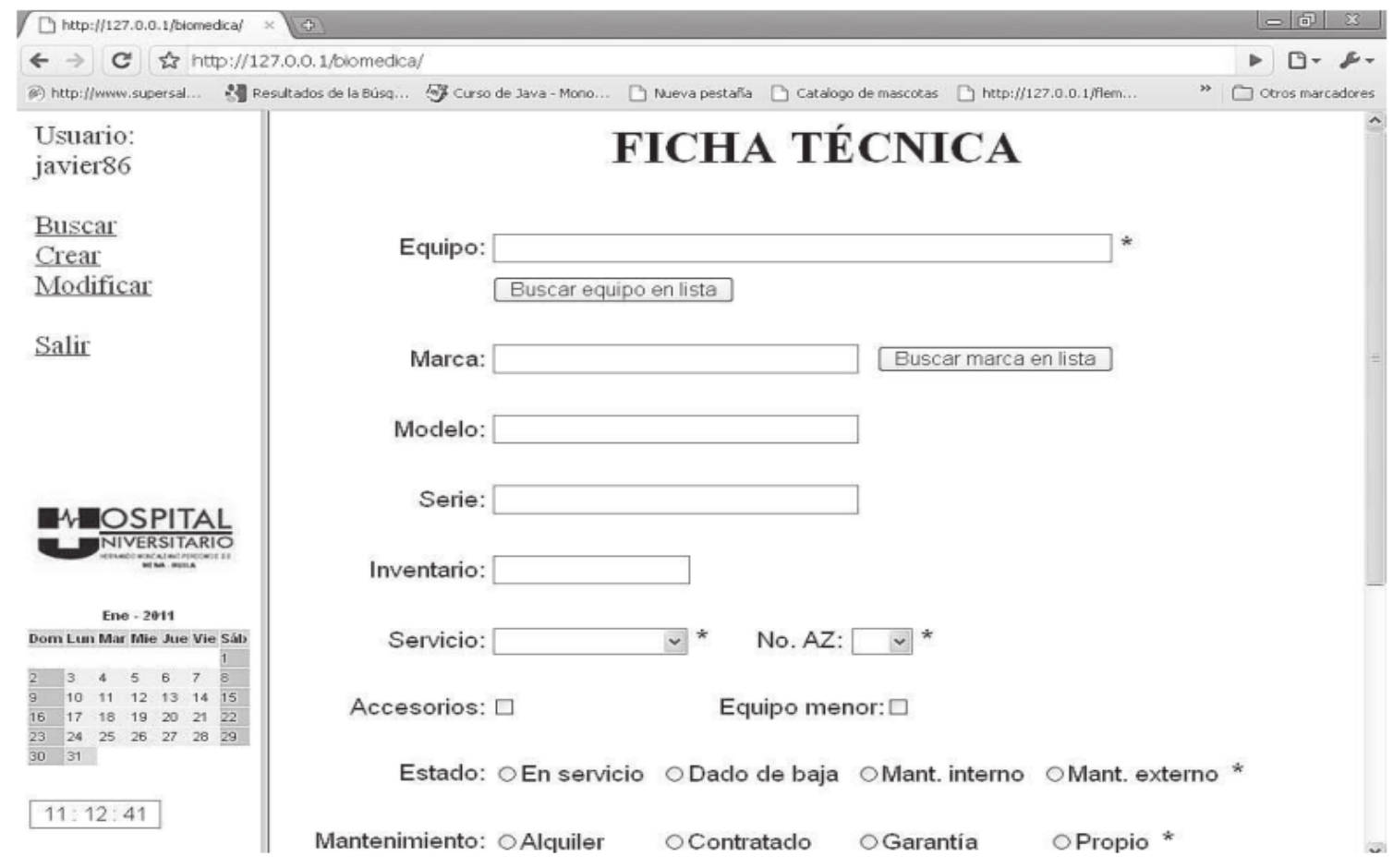

Figura 1. Interfaz visual con PHP

3.2Asignación de tags a los equipos: El proceso de asignación de tags a los equipos médicos, inicia primero por la información contenida de los equipos en la base de datos. Para esto se verifica si los datos del equipo existen, y sila información no se encuentra en la base de datos, se procede entonces a ingresar sus datos para almacenarlo y así generar su código interno. Luego, se procede a buscar el equipo desde la aplicación de C++ Builder (Figura 2), a través del código interno del equipo o mediante otros datos. Los datos del equipo seleccionado son mostrados alusuario, incluyendo el campo del UID del tag. Si el equipo tiene tag asignado, aparecerá su denominación en el campo de UID, y si no, el campo aparecerá vacío.

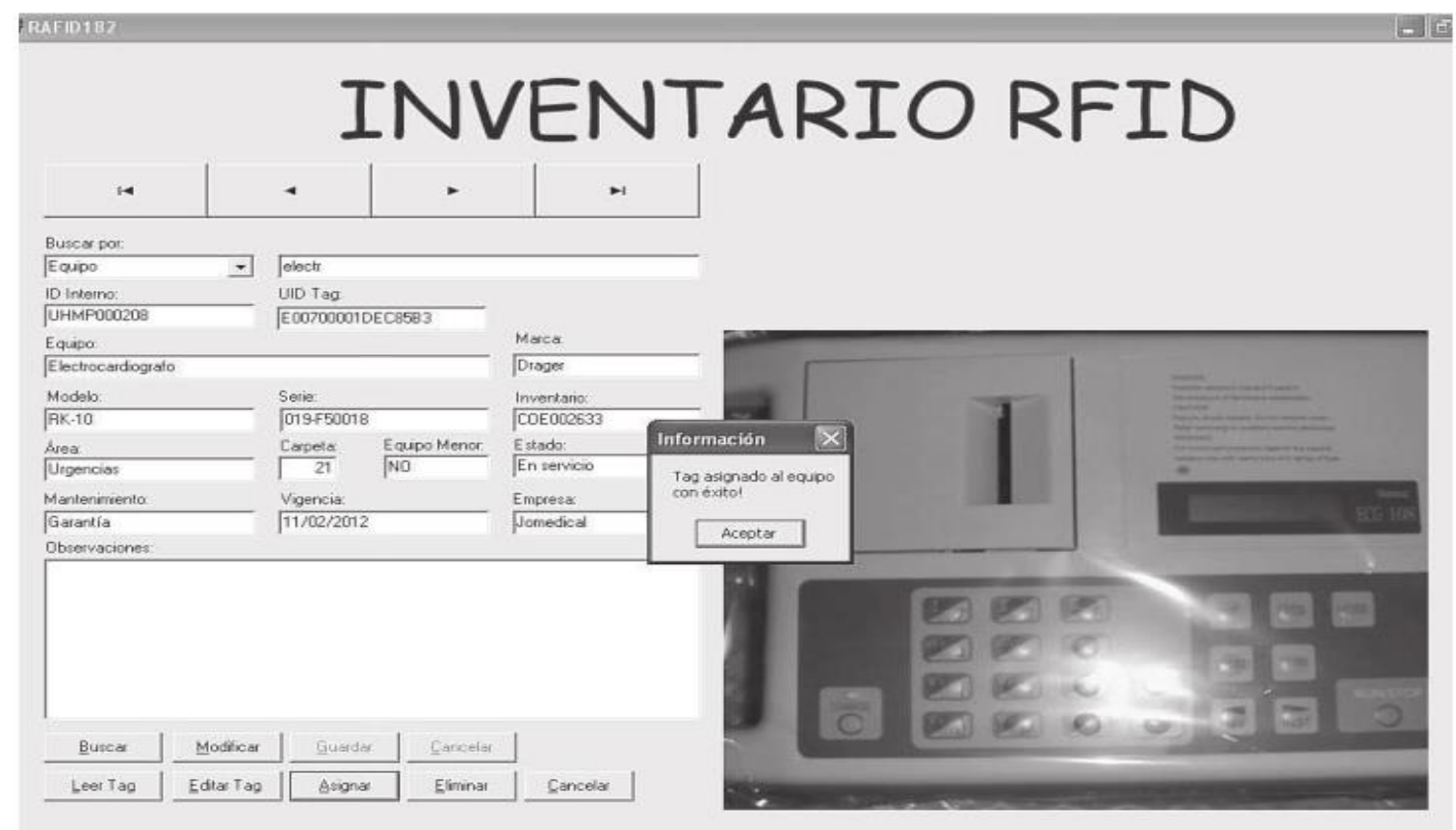

Figura 2. Interfaz visual con $\mathrm{C}++$ Builder 
Se procede a la lectura del tag que va a ser colocado en el equipo, ya con los datos seleccionados, en el cual se obtiene el UID. Se pueden realizar modificaciones de los datos antes, durante y después de la asignación del UID al equipo. El UID asignado queda relacionado de manera directa con el código interno del equipo de la base de datos, lo que permite con esto poder identificarlo a partir del uso de un lector RFID.

3.3 Identificación de los tags: La identificación se realiza mediante el uso del lector RFID. Cuando el tag se encuentre dentro de la zona de la antena del lector, automáticamente podrá ser reconocido por la aplicación de C++Builder a través de su UID, para luego mostrar la información respectiva.

Pensando en una posible utilización como sistema antirrobo con los propios tags RFID, se desarrolló una aplicación para este propósito (Figura 3). Para este caso el lector incorpora la función anticolisión para leer múltiples tags. El lector a través de su antena se dispone a leer los tags que se encuentren en su área de influencia. Si uno o más tags son detectados, se activa una señal auditiva y el sistema mostrará el listado de esos tags.

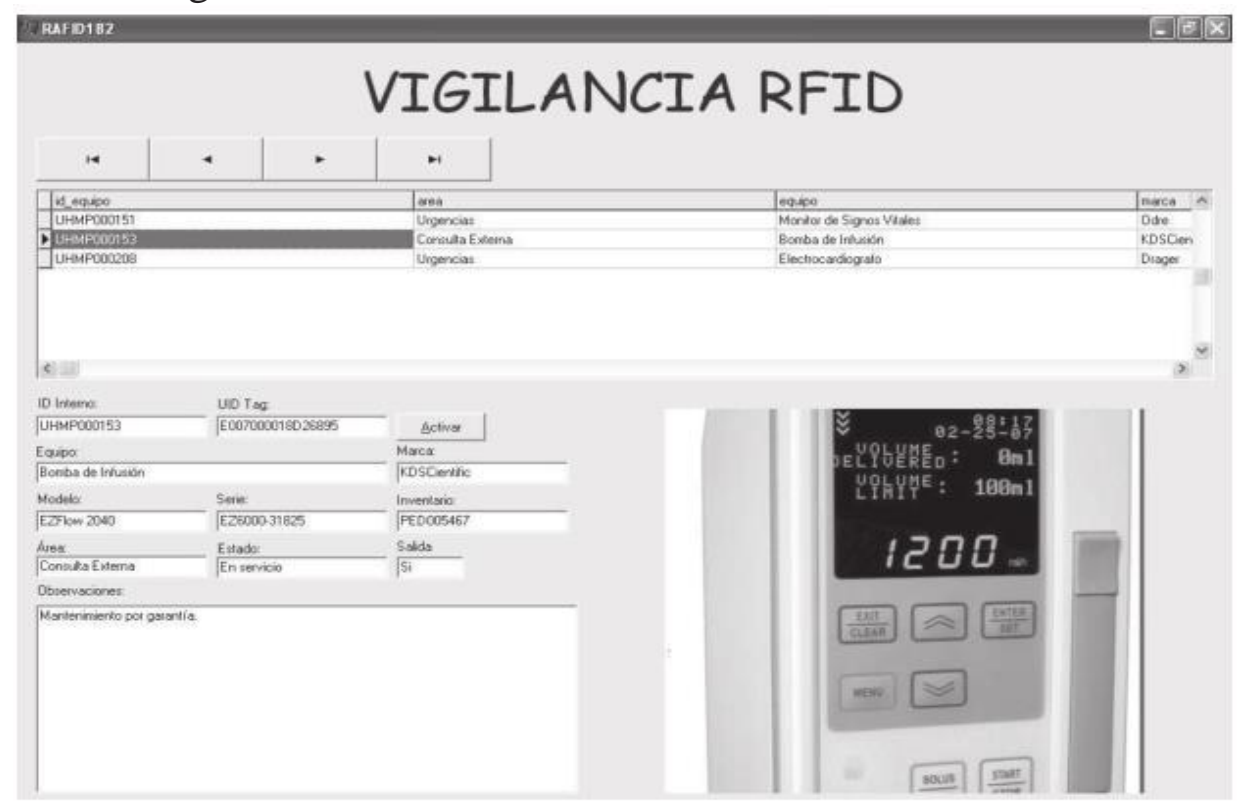

Figura 3.Lectura simultanea de 3 tags

3.4Resultados obtenidos del prototipo: Se realizaron pruebas para comprobar el comportamiento del lector ante un evento de lectura de múltiples tags, en el cual el lector aplica la función anticolisión. Además de lo anterior, verificar la conexión alabase de datos del servidor, por parte dela aplicación del lector.

La prueba consistió en tomar 3 tags y asignarlos a los datos de 3 equipos (Tabla 4), para luego obtener los datos del UID de cada tag con el lector, y a partir de cada UID mostrar en pantalla (Figura 3) la información de los equipos que se relaciona con esos tags. Para determinar el margen de éxito de la lectura del UID de cada tag, se procedió a realizar la lectura simultánea delos 3 tags, para lo cual se tuvo en cuenta la orientación en grados de los tags, respecto a la posición del lector. Se realizaron 4 lecturas para cada una de las orientaciones, obteniendo los resultados mostrados en las Tabla 5. Cabe notar que P1, P2, P3 y P4 son cada una de las pruebas de lectura simultánea realizadas a los tags, donde se coloca "Sí" a los tags reconocidos por el lector y "No" a los que no detecto el lector. Luego se determinó la probabilidad de éxito que tuvo cada tag, al ser reconocido por el lector y su aplicación.

Tabla 4. Listado de los equipos con sus respectivos UID

\begin{tabular}{|c|c|c|c|}
\hline UID Tag & ID Interno & Equipo & Servicio \\
\hline E007000018D26895 & UHMP000153 & Bomba de Infusión & Consulta Externa \\
\hline E00700001DEC85B2 & UHMP000151 & Monitor de signos vitales & Urgencias \\
\hline E00700001DEC85B3 & UHMP000208 & Electrocardiógrafo & Urgencias \\
\hline
\end{tabular}

Facultad deIngeniería Universidad Surcolombiana

Tabla 5.Resultados de la lectura simultánea de 3 tags 


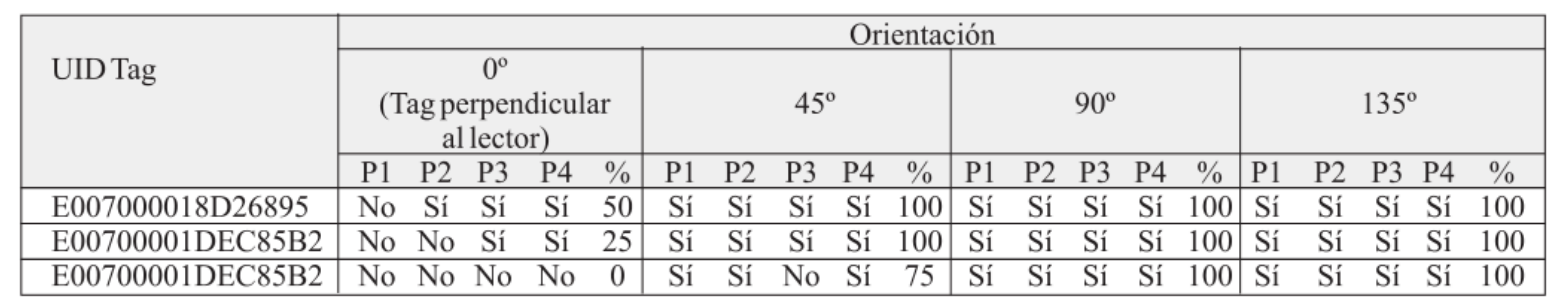

3. 5 Diseño del sistema RFID para el hospital: El sistema RFID para el hospital, se compone de 2 estructuras: la encargada de la gestión de los equipos médicos y la de seguridad antirrobo usando los propios tag.

En la figura 4, se puede observar el esquema planteado para gestionar los equipos médicos con los tags, se compone de un lector de mano con antena incorporada y un computador que tendrá la aplicación del lector, con el cual se podrá leer y administrar la información relacionada con los tags de los equipos, que residirá en la base de datos de un servidor.

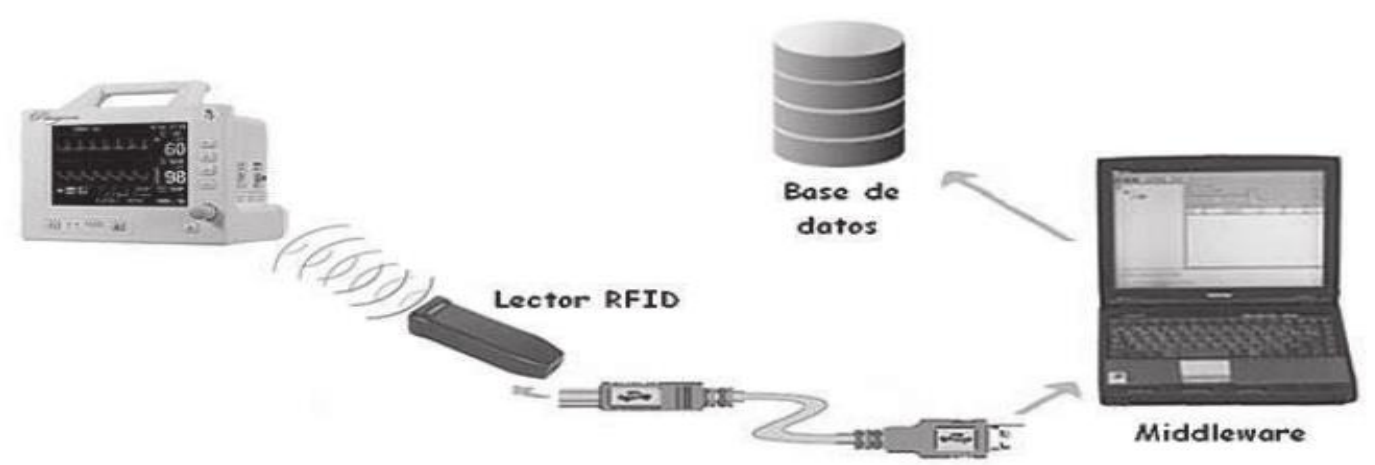

Figura 4. Esquema del sistema RFID para la gestión de los equipos

Se emplearan lectores portátiles para el inventario los equipos con los propios tags, en el cual el lector a través de su memoria interna almacenará el UID de los tags. De esta forma al realizar la lectura de los tags delos equipos, esta información podrá ser descargada del lector aun computador, para luego verificar y comparar los UID obtenidos por el lector, con los existentes en la base de datos. Esto permitirá reducir el error humano, que se presenta al realizar de forma manual el inventario de los equipos.

Para implementar el sistema RFID como sistema antirrobo, se tuvo en cuenta como primera medida el número de accesos con que cuenta al edificio del hospital (Principal, Consulta Externa y Urgencias), determinando así la clase y la cantidad de dispositivos para el sistema. En el diseño de este esquema (Figura S), se tuvo en cuenta el alcance de lectura para los tags, el tipo de antena, el modo de operación del lector y de los dispositivos en conjunto.

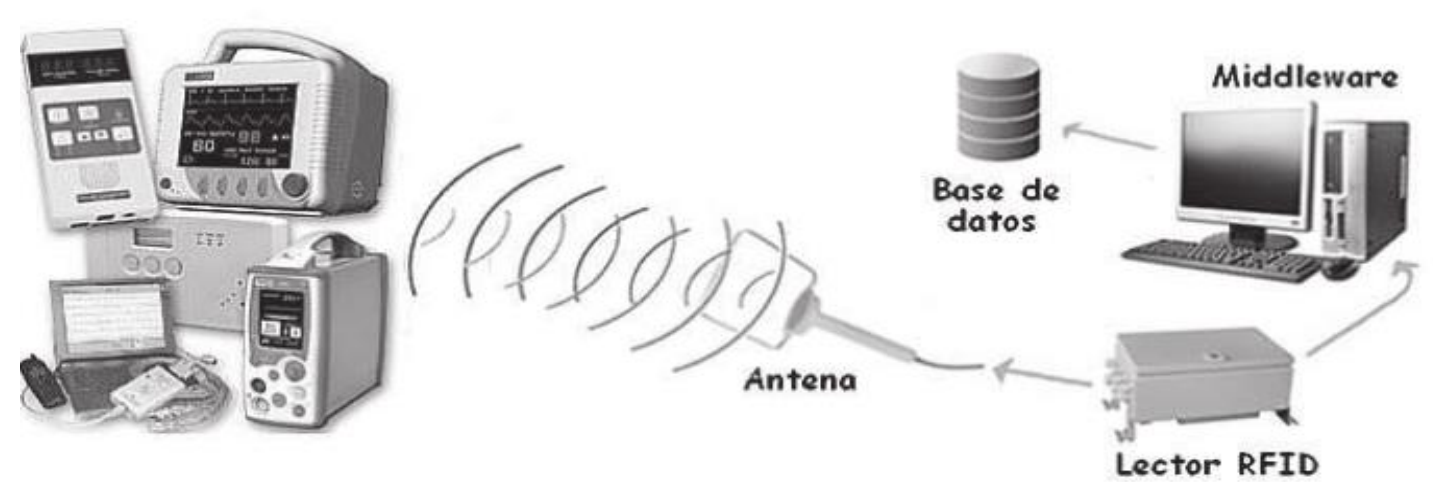

Figura 5. Esquema del sistema antirrobo con RFID para un acceso 
computador que contendrá el programa de aplicación para el lector, permitiendo también visualizar en pantalla los elementos que contienen adheridos los tags. El sistema RFID en cada acceso contará con un punto de enlace a red, para la conexión con la base de datos del servidor, que sedará a través dela aplicación del lector.

\subsection{Componentes propuestos para el sistema RFID del hospital:}

a).Lector RFID DTR14: Este lector de la empresa Ericel \& Detec Ingeniería (Ericel, 2010), se suministra con el software de administración del lector modificado, acorde con las peticiones del tipo de aplicación requerida. El dispositivo es de corto alcance y opera bajo el protocolo ISO 15693.Funciona con interfaz RS-232 y opera también como un lector portátil.

\section{Características:}

$\checkmark$ Distancia de lectura de tags hasta $5 \mathrm{~cm}$.

$\checkmark$ Almacenamiento del númerode identificación y datosescritos en el tag.

$\checkmark$ Todonuevo evento incluye hora y fecha dela lectura del tag.

$\checkmark$ Almacenamiento cíclicode los últimos 16.384 eventos de 32 caracteres cada uno.

$\checkmark$ Batería recargable de LITIO-ION de 3.6V.

$\checkmark$ Muy bajo consumo de energía con apagado automático después de las lecturas.

$\checkmark$ Lucesverde y rojoparainformación visual y señal audible.

$\checkmark$ Conexión al PC serial RS232 con velocidad programable entre 9600 y 57600 baudios.

b). Lector RFID RI-STU-655A: Este dispositivo de la compañía Texas Instruments (TEXAS, 2001), trabaja a una frecuencia de $13.56 \mathrm{MHz}$, el cual se propone para el uso del sistema antirrobo.

\section{Características:}

$\checkmark$ Capacidad para manejar 2 antenas

$\checkmark$ Potenciaconfigurable de hasta 10 -

$\checkmark$ Soporta estándares ISO 15693, Tag IT, EPC.

$\checkmark$ Lamáxima distancia de lectura/escritura que se logra es de 1 metro.

$\checkmark$ Ideal para aplicaciones de Access Control, Farmacéuticas, Bibliotecas, ID equipajes, identificación de ítems, elementosconlíquidos, etc.

$\checkmark$ Puertos de comunicaciones son configurables en RS232 y/o RS485.

c). Antena ISC.ANTH200/200-A: Esta antena portátil de mano de Texas Instruments (TEXAS, 2006), permite tener una gran área de lectura para facilitar el escaneo de tags, y es ideal para trabajar junto con el lector RI-STU-655A. Puede ser usado en gran variedad de lectores que trasmitan a una frecuencia de $1356 \mathrm{MHz}$ y que tengan una salida de impedancia de 50 ?. Con esta antena de mano se buscará reducir inconvenientes relacionados con la lectura delos tags, como el caso de la orientación o alcance de los mismos, de manera que el campo de lectura se pueda adaptar mejor a la posición de los tags.

\section{Características:}

$\checkmark$ Antena de mano con cableconexión.

$\checkmark$ Potencia configurable de hasta $2 \mathrm{~W}$.

$\checkmark$ Distancia máxima del cable $3.6 \mathrm{mts}$

d). Tag RI-I02-112B-03: Este tag de Texas Instruments (TEXAS, 2002) es compatible con el estándar ISO/IEC 15693. Ofrece al usuario la posibilidad de acceder a la memoria de 2048 bits del tag, para escribir y actualizar información adicional en ella. Contiene un único código identificador, el cual no puede ser alterado, garantizando la unicidad de cada tag. Por ser un tag pasivo y estar impreso en lámina de plástico, es bastante económico y puede ser colocadoen superficies planas. 
Características:

$\checkmark$ Compatible con estándares ISO/IEC 15693 eISO/IEC 18000-3

$\checkmark$ Frecuencia de operación de $13.56 \mathrm{MHz}$

$\checkmark$ Memoria de2048bits organizado en bloques de 64x 32 bits para escritura adicionalde datos.

e). Computador para la aplicación del sistema antirrobo: Se empleará un computador en cada acceso del hospital con el sistema antirrobo RFID, en donde correrá la aplicación de Builder $\mathrm{C}++$ para el lector y permitirá visualizar los equipos que se identifican a través delos tags, el cual se conectará a un punto de red para la conexión con el servidor.

\title{
Características:
}

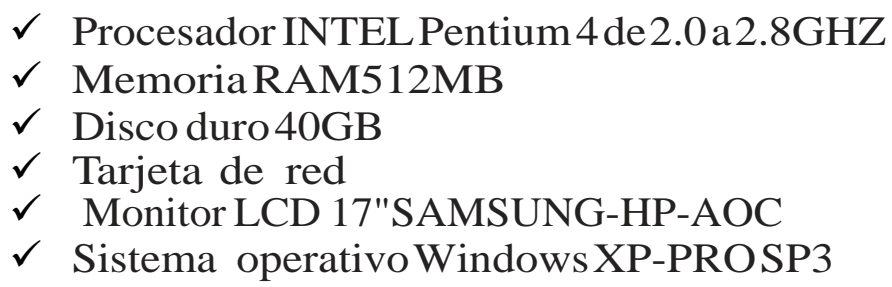

f). Servidor HP ProLiant ML110 G6: Este servidor de la empresa Hewlett Packard (Hewlett Packard, 2010) permitirá gestionar la información de la base de datos delos equipos médicos, con el cual se conectará cada una de las aplicaciones de los lectores RFID del hospital. En el correrá la aplicación realizada en PHP, para permitir al usuario hacer uso dela interfaz visual desde una conexión remota.

\section{Características:}

\author{
$\checkmark$ Procesador Intel Core i3 530/2.93 GHz \\ $\checkmark$ Disco duro de $160 \mathrm{~GB}$ \\ $\checkmark$ Sistema operativo Windows XPSP2
}

3.7 Limitaciones: Aunque la tecnología RFID en comparación con otras tecnologías de identificación automática, tiene como única limitante su costo, presenta interesantes ventajas y abre caminos para muchas aplicaciones, con la capacidad de garantizar la unicidad y evitar las falsificaciones de sus elementos identificativos.

\begin{abstract}
4. Conclusiones
De acuerdo a las pruebas realizadas con el prototipo (Tablas 5),para la aplicación como sistema antirrobo se puede presentar ciertas restricciones sobre su funcionamiento, debido a que la operatividad para este sistema se basa en cuestiones técnicas ofrecidas por los fabricantes de los dispositivos y a resultados logrados por sistemas similares de radiofrecuencia, como los que existen en los grandes almacenes de cadena, por lo que su aplicabilidadestá sujetoa la experimentación.
\end{abstract}

Uno de los inconvenientes en la propuesta del sistema RFID del hospital, fue la elección de la antena para el lector RFID del sistema antirrobo, pese a haber antenas tipo portal, con alcance de lectura entre 1.3 y hasta 2 metros, se optó por una antena móvil, debido a que con este tipo de antena hay más adaptabilidad a la posición de lectura del tag, evitando así los problemas que se puedan presentar con la orientación de estos (Tablas 5).Además se tuvo en cuenta que generalmente hay un proceso de inspección por parte del personal de seguridad y por el costo elevado que presenta las antenas tipo portal.

Se presentó un inconveniente en el planteamiento del sistema antirrobo, ya que cada punto de acceso del hospital debería tener un sistema RFID, que a su vez estaría 
Conectada alabase de datos del servidor, por lo que se optó por colocar un computador junto con la aplicación del lector en cada acceso, para la conexión con el servidor.

a través de un punto de red interna y poder visualizar la información respectiva. Otro motivo fue porque el hospital cuenta con un cableado estructurado definido, por lo que no era conveniente hacer modificación alguna a esta red.

La importancia de este estudio de viabilidad ha demostrado relevancia, en cuanto a lo relacionado con el manejo de la información, por lo que el personal del departamento de biomédica ha demostrado gran interés en la aplicación del software para la administración de la información, en parte por la seguridad que ofrece su almacenamiento en una base de datos y por la facilidad en el manejo que la interfaz en PHP ofrece. Debido a esto, se ha pensado hacer una prueba piloto con la información real de los equipos, buscando así en el futuro realizar las modificaciones y mejoras para dejar en funcionamiento.

\section{Referencias Bibliográficas}

1.Attaran, Mohsen., 2006. Emerging Signs of an RFID Payoff. Consultado el 10 de
mayo

2. Engelschall, Ralf S., 2001. Apache Desktop Reference, Adobe PDF Document. URL: http://www.apacheref.com/book/adr.pdf

3. Ericel \& Detec Ingeniería, 201O. Sistema de control de ruta EVIDENCE. Consultado el 14 de agosto de 201O. http://www.angelfire.com/co3/bog/M_ADEvidence.html

4. Ericel \& Detec Ingeniería, 2009. Lector DTR.10 Guía de referencia, Adobe PDF Document. URL: http://wwwangelfire.com/co3/bog/pdf/READER_DTR 10 pdf

5. Hewlett Packard, 2010. HP ProLiant MLllO G6 server. Consultado el 18 de septiembre de 2010. http://h18004.wwwl.hp.com/products/quickspecs/DS_00178/DS_00178. pdf

6. Portillo García, Javier 1., Bermejo Nieto, Ana B., Bernardos Barbolla, Ana M., 2008. Informe de Vigilancia Tecnológica madri+d ${ }^{11}$ Tecnología de identificación por radiofrecuencia (RFID): Aplicaciones en el ámbito dela salud". Fundación madri+d para el Conocimiento, Madrid, España. 176p.

7. RFID Journal, 201O. Hospital utiliza tecnología RFID para la gestión de activos InHouse. Consultado el 29 de junio de 2010. http://www.rfidpoint.com/noticias/hospital-utilizatecnologia-rfid-para-la-gestion-de- activos-in-house/

8. TEXAS INSTRUMENTS, 2006. Advanced reader technologies i-scan HF (13.56 $\mathrm{MHz}$, Adobe PDF Document. URL: http://www.cipam.com/images/telechargement/Public /anth200_200_e.pdf?PHPSESSID=8f lb9b248e6a672eabe6ff:ff6b4af897

$\begin{array}{lcccc}\text { 9. TEXAS } & \text { INSTRUMENTS, 2004. RI-117-112A-03Tag-itTM } & \text { HF-IPLUS } \\ \text { TRANSPONDER } & \text { INLAYS CD, } & \text { Adobe PDF } & \text { Document. } & \text { URL:http: } \\ \text { //www.ti.com/rfid/docs/manuals/pdfSpecs/RI-I17-1 } & \text { 12A-03.pdf } & & \end{array}$

10. TEXAS INSTRUMENTS, 2002. Tag-it_ HF-I Transponder Inlay RectangleLarge, Adobe PDF Document. URL:http://www.youwokeji.com.cn/down/RI-I02-1 12B.pdf

11. TEXAS INSTRUMENTS, 2001. HF Reader System Series 6000 S6550 Long Range Reader (Housed), Adobe PDF Document. URL:http//www.ti.com/rfid/docs /manuals/pdf Specs/RI-STU-655AdataSheet.pdf

12. Valade, Janet., 2004. PHP y MySQL para Dummies. ST Editorial. Edición 2da. México D.F., México.438páginas 